\title{
What do measurements of molecular biomarkers in different body fluids really tell us?
}

\author{
A Robin Poole* \\ See related research by Catterall et al., http://arthritis-research.com/content/12/6/R229
}

\begin{abstract}
Molecular or biochemical biomarkers of joint metabolism offer promise in helping us understand joint pathology, its detection and treatment. But they have often been studied alone and in only one body fluid. Although the synovial joint is usually the focus of most arthritis pathology, it is often difficult, for a variety of reasons, to obtain synovial fluid that should best reflect changes in biomarkers related to pathology. It is therefore very important to see whether analyses of more readily obtainable sera and urine also reflect changes in synovial fluid. Catterall and colleagues, in a paper in Arthritis Research \& Therapy that examines very early biomarker changes following joint injury, provide us with some insights into these important questions. As the study was very small and examined very early changes following joint injury, prior to onset of any recognisable pathology, we look forward to future larger biomarker studies of this kind in patients with clinically defined arthritic changes to which we can relate biomarker data.
\end{abstract}

The ability to accurately monitor changes in joint metabolism in vivo that may lead to and predict, at a much later date, onset of structural pathology detected by imaging is an important challenge for osteoarthritis (OA) biomarker research. A recent study has examined very early molecular biomarker changes in synovial fluid (SF), sera and urine following anterior cruciate ligament rupture [1]. This damage often leads, many years later, to the onset of OA. In animals, such an injury can result in OA onset within weeks [2]. As the joint is considered the focus of the pathology, it is important to determine whether analyses of more accessible body fluids (urine

*Correspondence: a.poole@mcgill.ca

Department of Surgery, 687 Pine Avenue West, McGill University, Montreal, Quebec H3A 1A1, Canada and serum) reflect biomarker changes in OA joints. SF, serum and urine samples were taken from 11 patients on two consecutive occasions approximately 15 and 47 days after rupture. Twenty-one different biomarkers were analysed but seven were restricted to SF measures only. Previously, most biomarker studies - with some exceptions [3] - examined one or two biomarkers of special interest and availability, often in only one body fluid. The study of Catterall and colleagues is therefore helpful in that it sought to gain a broader picture of biomarker changes and their interrelationships between fluids [1].

The declines in SF proteoglycan glycosaminoglycan and aggrecan cleavage neoepitope contents contrasted with an increase in biomarkers of type I collagen degradation, namely CTx1, NTx, C1,2C (the latter also detects type II collagen cleavage), and the cartilage type II collagen cleavage biomarker CTxII, now known to be mainly generated by type II cleavage in calcified cartilage [4]. But the authors' claims that these proteoglycan and collagen changes reflect what is seen in vitro when cartilage degradation is stimulated [1] are debateable, particularly since the most significant collagen changes involved mainly type I collagen. One must also question the conclusion that these biomarkers 'demonstrated that there is significant and measurable cartilage and bone damage after acute knee injury'. No such changes were shown clinically.

Some of the most interesting data in this paper come from asking whether biomarkers in body fluids reflect primarily joint-derived sources. The much increased contents in SF over serum of aggrecan FA846, COMP and MMP3 point to a joint origin for these biomarkers. But there is only a correlation between serum and SF for MMP3, and then it is rather weak. It may therefore be better to look at these biomarkers in SF rather than in serum.

In contrast, the serum/urine bone biomarkers CTx 1 , NTx and osteocalcin show strong correlations with SF. None of these biomarkers are really elevated in SF (1.6-fold, 1.2-fold and 1.2-fold, respectively), however, suggesting that they arise primarily systemically, thereby explaining these correlations. If the biomarkers do arise 
systematically, then this would indicate more systemic changes in bone metabolism. Uninjured and nonarthritic controls are needed to help answer such questions.

An analysis of the interrelationships and possible correlations between bone, cartilage and inflammation metabolism reflected by biomarkers is invariably useful but was overlooked. Not only are the effects of inflammation important to understand, but also whether bone changes accompany early changes in cartilages as suggested above. Analyses of data for ratios between biomarkers, such as those of matrix turnover/synthesis and degradation, were also lacking. These insights into the balance between synthesis and degradation can provide valuable additional information $[5,6]$.

Earlier work by Kraus and colleagues pointed to the importance of relating measurements in SF to urea to correct for dilutions caused by joint effusions [7]. Yet this correction was not applied by Catterall and colleagues. Are such corrections not necessary?

The present discussion of this study by Catterall and colleagues [1] has provided important but sobering insights and reminders - we should exercise great caution in how we interpret biomarker data, and should endeavour to make sure we understand what the data mean. Until we have definitive indications that given changes in biomarkers in a given body fluid do indeed consistently reflect specific clinical changes, we must avoid putting any reliance on biomarker data alone. We are still in an exploratory/assessment phase in our understanding of what molecular biomarkers can really offer us. We have often seen that measurements in sera or urine may have no relationship to events measured in joints. The study population was very small, asking too much of statistical analyses, and often only SFs were examined [1]. Studies were made of early events long before any degenerative structural changes would be expected, to which we could relate and make sense of the biomarker data. What we need are future clinical studies assessing head to head many different biomarkers and different body fluids, as in this investigation. But in addition we must have structural joint changes in bone and cartilage to which we can relate.
Biomarker analyses alone are no longer the way to go. Thankfully the private/public OA initiative launched by the NIAMS/NIH and industry - now involving the expertise offered by the Osteoarthritis Research Society International - is one exercise that should provide us with momentum in better understanding biomarkers of different kinds.

Abbreviations

OA, osteoarthritis; SF, synovial fluid.

Competing interests

ARP is a consultant to IBEX, Montreal, Canada.

Published: 27 April 2011

References

1. Catterall JB, Stabler TS, Flannery CR, Kraus VB: Changes in serum and synovial fluid biomarkers after acute injury. Arthritis Res Ther 2010, 12:R229.

2. Poole AR, Blake S, Buschmann M, Goldring S, Laverty S, Lockwood S, Matyas J, McDougall J, Pritzker K, Rudolphi K, van den Berg W, Yaksh T:

Recommendations for the use of preclinical models in the study and treatment of osteoarthritis. Osteoarthritis Cartilage 2010, 18:S10-S16.

3. Cibere J, Zhang H, Garnero P, Poole AR, Lobanok T, King L, Saxne T, Kraus VB, Way A, Thorne A, Wong H, Singer J, Kopec J, Guermazi A, Peterfy C, Nicolaou C, Munk P, Esdaile JM: Association of biomarkers with pre-radiographically defined and radiographically defined knee osteoarthritis in a populationbased cohort. Arthritis Rheum 2009, 60:1372-1380.

4. Bay-Jensen AC, Andersen TL, Charni-Ben Tabassi N, Kristensen PW, Kjaersgaard-Andersen P, Sandell L, Garnero P, Delaisse JM: Biochemical markers of type II collagen breakdown and synthesis are positioned at specific sites in human osteoarthritic knee cartilage. Osteoarthritis Cartilage 2008, 16:615-623.

5. Cahue S, Sharma L, Dunlop D, lonescu M, Song J, Lobanok T, King L, Poole AR: The ratio of type II collagen breakdown to synthesis and its relationship with the progression of knee osteoarthritis. Osteoarthritis Cartilage 2007, 15:819-823.

6. Garnero P, Ayral X, Rousseau JC, Christgau S, Sandell L, Dougados M, Delmas PD: Uncoupling of type II collagen synthesis and degradation predicts progression of joint damage in patients with knee osteoarthritis. Arthritis Rheum 2002, 46:2613-2624.

7. Kraus VB, Huebner JL, Fink C, King JB, Brown S, Vail TP, Guilak F: Urea as a passive transport marker for arthritis biomarker studies. Arthritis Rheum 2002, 46:420-427.

doi:10.1186/ar3276

Cite this article as: Poole AR: What do measurements of molecular biomarkers in different body fluids really tell us? Arthritis Research \& Therapy 2011, 13:110. 